\title{
FLEXIBLE IMPEDANCE SENSOR FOR WIRELESS MONITORING OF CATHETER BIOFILMS
}

\author{
R.C. Huiszoon ${ }^{1,3}$, J.M. Stine ${ }^{1,2}$, L.A. Beardslee ${ }^{1}$, \\ P. Ramiah Rajasekaran ${ }^{1}$, W.E. Bentley, and R. Ghodssi, ${ }^{1,2,3 *}$ \\ ${ }^{1}$ Institute for Systems Research, ${ }^{2}$ Department of Electrical and Computer Engineering, \\ ${ }^{3}$ Fischell Department of Bioengineering \\ University of Maryland, College Park, Maryland, USA
}

\begin{abstract}
A microfabricated flexible impedance sensor (FlexIS) for wireless monitoring of bacterial biofilm formation on catheters is presented. The system consists of interdigitated electrodes on flexible polyimide film, interfaced with the cylindrical surface of a urinary catheter. An AD5933 impedance converter (AIC) measures sensor impedance and enables wireless readout by communicating with a smartphone via Bluetooth. A 5\% decrease in impedance at 1 $\mathrm{kHz}$ corresponded to the growth of an Escherichia coli biofilm for 24 hours on the catheter surface, compared to an increase of $2 \%$ for an unseeded control. Measurement results from the AIC and a standard potentiostat were compared, showing a similar decrease in impedance with biofilm formation. Furthermore, the sensor response to biofilm formation was supported by measured changes in biofilm biomass. The FlexIS allows in situ biofilm characterization in complex and inaccessible environments in clinical and research settings.
\end{abstract}

\section{INTRODUCTION}

Biofilms are the primary mode of growth for many bacteria, where planktonic cells adhere to hydrated surfaces and encase themselves in a sticky, polysaccharide matrix [1]. The formation of biofilm is driven by intercellular communication via small molecules, in a process known as quorum sensing [2]. The biofilm phenotype affords the constituent bacteria increased tolerance to antibiotic therapy, requiring doses 50-500 times larger for complete killing compared to their planktonic counterparts [3]. Analogous to metastasis, biofilms can serve as a source of recurring infection, as cells slough off of mature biofilms, allowing the bacteria to spread and colonize other areas. Therefore, there is potential risk of chronic infection if the source biofilm is not eliminated. Biofilm colonization accounts for a significant portion of hospital-acquired infections, especially in patients with implanted or inserted medical devices. Notably, urinary catheters cause bacteriuria at a rate of $5-7 \%$ per day of implantation due to biofilm formation [4]. There is currently a lack of adequate tools for detecting biofilm formation on medical devices. Care providers typically rely on time-consuming and labor-intensive plate culture and colony counting methods to identify bacterial infections [5]. Furthermore, this quantifies only the viable cells, not the presence of biofilm. In research settings biofilms are often characterized by optical techniques which rely on end-point staining and bulky equipment [6-7]; this does not lend itself to in situ application on the complex surfaces where biofilms form.

A limited number of device-based approaches have been developed to address the challenges associated with characterizing and detecting biofilm formation. Mechanical, optical, and electrical microsensors have been investigated for real-time biofilm sensing. Optical approaches using charge coupled devices (CCDs) to sense biofilm via optical density changes have been explored. Mechanical microsensors have been investigated as well, including quartz tuning forks, quartz crystal microbalances, and surface acoustic wave sensors, which rely on the accumulation of mass at the sensor surface [9-11]. However, these rely on rigid crystalline materials which introduce packaging constraints when integrated with complex, 3D surfaces for in situ operation. Electrical sensors utilizing electrodes to detect biofilm via change in electrical or electrochemical properties have also shown promise. Several groups have been able to detect biofilm formation by monitoring the impedance of electrodes in a rigid, planar setting [12-14]. However, these microfabricated impedance sensors are not amenable to integration with the complex 3D surfaces that are vulnerable to biofilm. To overcome this challenge, our group has recently demonstrated a flexible electrode platform for detecting biofilm formation in real-time using impedance measured with a benchtop potentiostat [15-16]. This work enables wireless in situ biofilm monitoring using the AIC, which can be interfaced with a Bluetooth microcontroller. FlexIS allows the degree of biofilm formation to be identified quickly and non-invasively, enabling effective treatment (Figure 1).
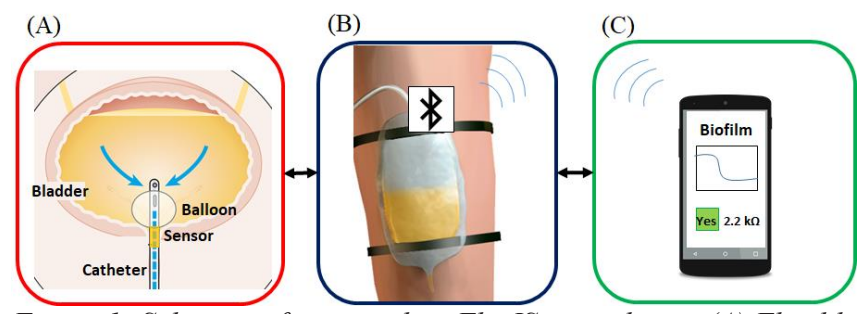

Figure 1: Schematic for a wireless FlexIS on catheter: (A) Flexible sensor embedded in urinary catheter; (B) Wireless electronics module attached to external catheter bag; (C) Smartphone app provides an alert in the presence of biofilm and allows prompt implementation of treatment.

\section{MATERIALS AND METHODS}

\section{System Fabrication}

The flexible impedance sensor is comprised of thin-film interdigitated electrodes (IDEs, $\mathrm{Cr}(20 \mathrm{~nm}) / \mathrm{Au}(200 \mathrm{~nm})$ ) patterned on a $25.4 \mu \mathrm{m}$-thick polyimide substrate in a lift-off process. This process consists of affixing a piece of the polyimide (Kapton, DuPont) onto a four-inch $\mathrm{Si}$ wafer via heat-release tape (Semiconductor Equipment Corp.) followed by patterning of electrodes using a standard photolithography process with Shipley 1813 resist (Microchem). Each wafer was exposed to $\mathrm{O}_{2}$ plasma for 1 minute at $200 \mathrm{~W}$ at $100 \mathrm{sccm}$ to improve metal adhesion on the polyimide. The electrodes were deposited via e-beam evaporation (Angstrom Engineering). Electrode fabrication was completed by lift-off in acetone for 1 minute. The polyimide film with the devices was separated from the wafer by heating to $150{ }^{\circ} \mathrm{C}$, prompting release by the tape. The devices, five per wafer, were cut into each die with scissors to complete fabrication. The final footprint of the IDEs was $10 \mathrm{~mm}$ x $40 \mathrm{~mm}$, connected to contact pads by $30 \mathrm{~mm}$ leads. The IDE width and spacing was $300 \mu \mathrm{m}$. The sensor was integrated with the interior lumen of an $11 \mathrm{~cm}$-long section of a 16 Fr silicone catheter (Allegro Medical) for testing. Uncured 
polydimethylsiloxane (PDMS) (Dow-Corning) mixed at a ratio of 10:1 base to curing agent was coated on the interior of the catheter tube by introducing a large drop at the inlet, and spreading it over the entire surface with a wooden applicator rod. The flexible device was introduced while the surface was coated in uncured PDMS. Then, the PDMS was cured for $3 \mathrm{~h}$ at $60^{\circ} \mathrm{C}$ to fix the polyimide substrate in place. The device was connected to either a potentiostat or an electronic module including the AIC. The AIC can be programmed via a BGM121 Bluetooth microcontroller to run a frequency sweep and display the data on a computer terminal or smartphone app. The AIC and BGM121 can be combined in a wireless electronics module contained in a custom 3D-printed housing, with clasps to hold it in place at the base of the catheter near the connection to the catheter bag. Figure 2 shows the device adhered in a catheter, interfaced with the electronics for wireless communication in the custom 3D-printed housing.
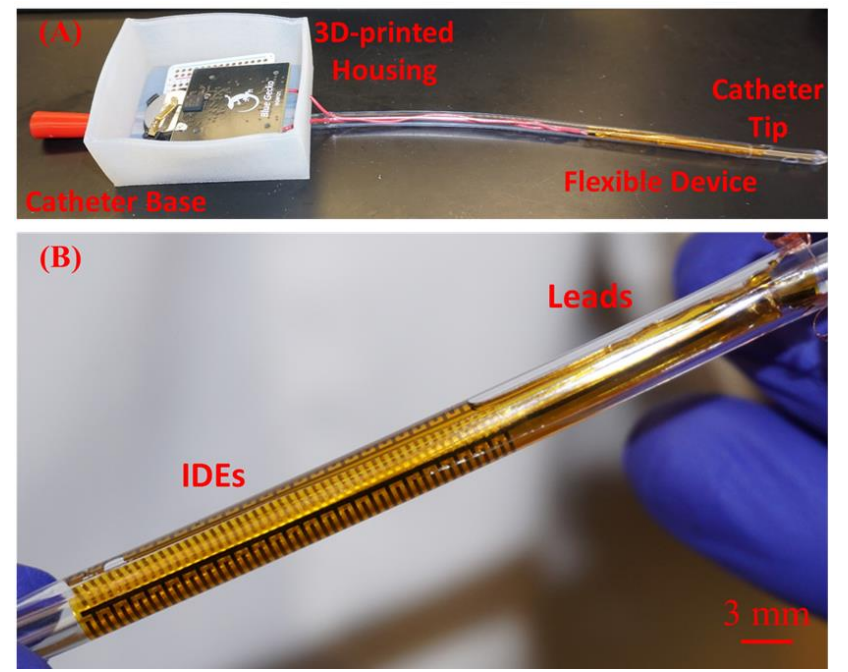

Figure 2: Optical images of the (A) urinary catheter with device integrated on the interior near the tip, connected to the AIC (under $B G M 121)$ and BGM 121 Bluetooth microcontroller contained in a 3D-printed housing (printed with Agilus30) which is affixed to the base of the catheter and (B) close-up image of inserted device.

\section{Device Testing}

The flexible impedance sensor was characterized using a custom flow system consisting of a growth media reservoir, waste reservoir, and the device connected using tygon tubing and luer connectors (Cole-Parmer) in between the reservoirs. The entire system is sterilized for 45 minutes in an autoclave at $121^{\circ} \mathrm{C}$ prior to the experiment, to prevent contamination by environmental microbes. The flow is driven by a peristaltic pump (Elemental Scientific). The sensor and tubing are placed in an incubator maintained at $37^{\circ} \mathrm{C}$, to mimic the temperature found in an inserted catheter. Escherichia coli K12 W3110 are cultured from frozen samples for 20 hours in $5 \mathrm{ml}$ of Luria broth (LB) media (Sigma) in an Innova 4000 incubator shaker at $37{ }^{\circ} \mathrm{C}$ (New Brunswick Scientific) set to $250 \mathrm{rpm}$. The cultured bacteria are then diluted to an $\mathrm{OD}_{600}$ of 0.25 , and $1 \mathrm{ml}$ of the diluted E. coli solution is introduced into the catheter tube directly via syringe. The bacteria are allowed to attach to the interior surface under static (no flow) conditions for 2 hours. This is referred to as the 'Seeding' phase. Immediately following Seeding, pure LB media is flowed at a constant flow rate of $7 \mathrm{ml} / \mathrm{h}$ for 24 hours. This is referred to as the 'Growth' phase. Throughout the Growth phase, the system impedance is measured by the AIC every 2 minutes. The relative change is used to monitor biofilm formation. Devices without any bacterial cells introduced were also tested as a control. This procedure was also performed using a benchtop potentiostat (Electrochemical Workstation 660D, CHInstruments) for the impedance measurements, to serve as a comparison standard for evaluating the efficacy of the system. Crystal violet (CV) absorbance staining is used to quantify the end-point biomass. This serves to validate the impedance measurements by correlating them with the increase in strongly adhered biomass. This process involves flowing the CV stain into the tube and allowing it to bind to the adhered proteins and DNA associated with the biofilm; thus, bound stain is proportional to biofilm biomass. Then, the sample is rinsed with deionized water to remove unbound stain. Finally, decomplexation solution ( $80 \%$ ethanol: $20 \%$ acetone) is introduced into the system for 30 minutes. The stain is soluble in decomplexation solution and dissolves into the solution. Optical absorbance at $\lambda=590 \mathrm{~nm}$ is measured, with increases corresponding to increased biomass. One-way analysis of variance (ANOVA) was used to determine statistical significance of the biomass quantification results.

\section{RESULTS AND DISCUSSION \\ Potentiostat Impedance Sensing}

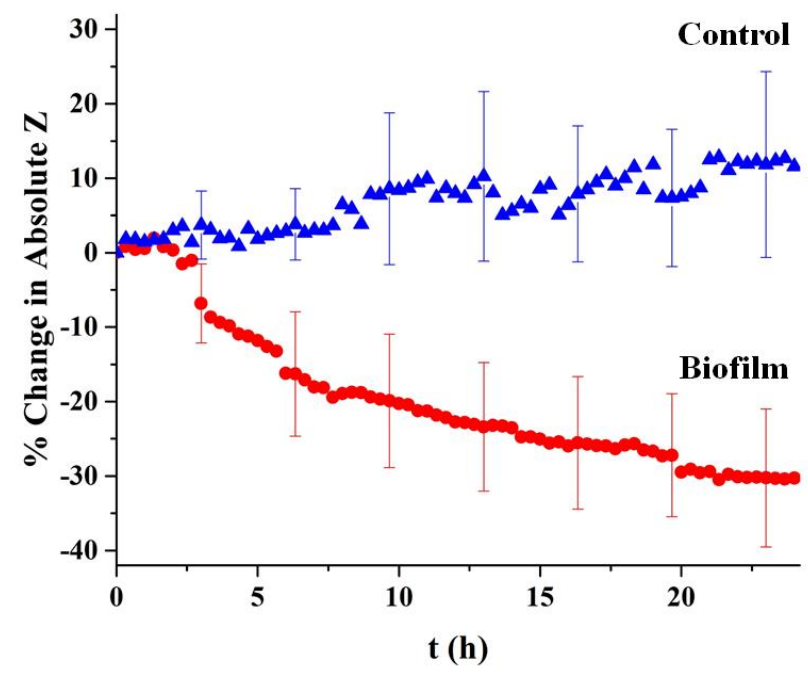

Figure 3: Average real-time impedance sensing results over the course of the 24-hour growth period, measured by the benchtop potentiostat. The blue triangles correspond to the control samples (without biofilm formation) and the red circles correspond to the samples with biofilm formation. The excitation signal used was $A C$ $50 \mathrm{mV}$ at $100 \mathrm{~Hz}$, chosen to optimize sensitivity. The error bars correspond to standard error $(N=5)$; the large standard error is related to the inherent variability in biofilm formation.

Figure 3 shows the real-time change in impedance measured with the benchtop potentiostat. In the first 2 hours, the signals for both samples show relatively little change. However, from hours two to three, the impedance of the biofilm sample abruptly decreases by $7.2 \%$. This is likely related to the rapid proliferation phase of biofilm growth. The impedance decrease slows after this initial drop; from hours three to 24 the impedance drops approximately $1 \%$ per hour. Over the course of the entire Growth phase the system impedance exhibits a dramatic $30 \%$ decrease. By comparison, the control sample exhibits a steady increase of about $10 \%$ over the entire growth phase. The impedance decrease is due to 
the accumulation of charged proteins and ions on the sensor surface associated with the biofilm and biofilm metabolism causing a shift in the double layer capacitance [12],[17].

The resulting end-point biomass can be compared between samples with and without biofilm, and the trend can be compared with the impedance sensing results. The biomass quantification results for samples measured with the potentiostat are shown in Figure 4. The absorbance nearly doubled on average in samples with biofilm compared to the biofilm-free controls. The control signal represents the contribution from the LB media alone. The increased absorbance associated with biofilm was statistically significant according to one-way ANOVA $(\mathrm{p}<0.05)$, confirming the increase in biomass. Furthermore, this increase in biomass is correlated with the decrease in impedance measured with the flexible sensor.

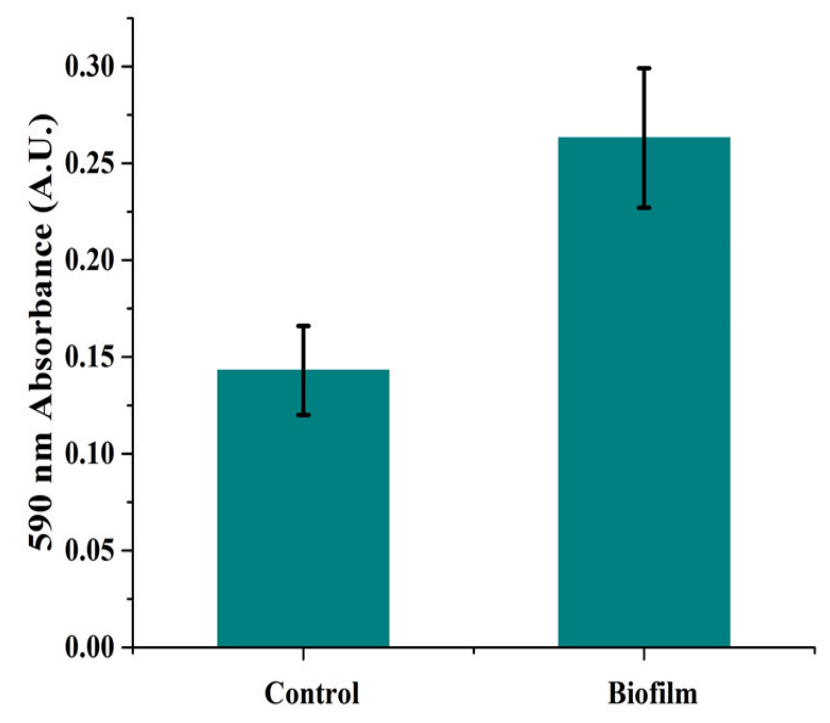

Figure 4: End-point biomass quantification for samples with and without biofilm. Error bars correspond to standard error $(N=3)$.

\section{AIC Impedance Sensing}

The real-time impedance sensing results from samples measured with the AIC are presented in Figure 5. The AIC measures a gradual decrease of approximately 5\% after 24 hours of biofilm growth. The biofilm-free samples, by comparison, showed a slight increase of about $2 \%$ in impedance after 24 hours. This trend corresponds with the results obtained using the FlexIS with a standard benchtop potentiostat. Both measurement approaches indicate that a significant decrease in system impedance occurs with biofilm formation. In addition, they both show a slight increase in the control samples. While the trends were similar between the two approaches, the magnitudes of the changes were different; a significantly smaller impedance shift when measured by the AIC. This decreased sensitivity is attributed to the differences in how each system is calibrated. Additional electronic circuitry may be necessary to further improve the FlexIS sensitivity when using the AIC for wireless biofilm monitoring. The similar trends between each approach suggest that using the FlexIS with the AIC is a viable real-time wireless biofilm sensing tool.

A CV stain was also performed to quantify the end-point biomass for samples measured with the AIC, to correlate the change in impedance with an increase in biomass from biofilm. The biomass quantification results for samples measured with the AIC are shown in Figure 6.

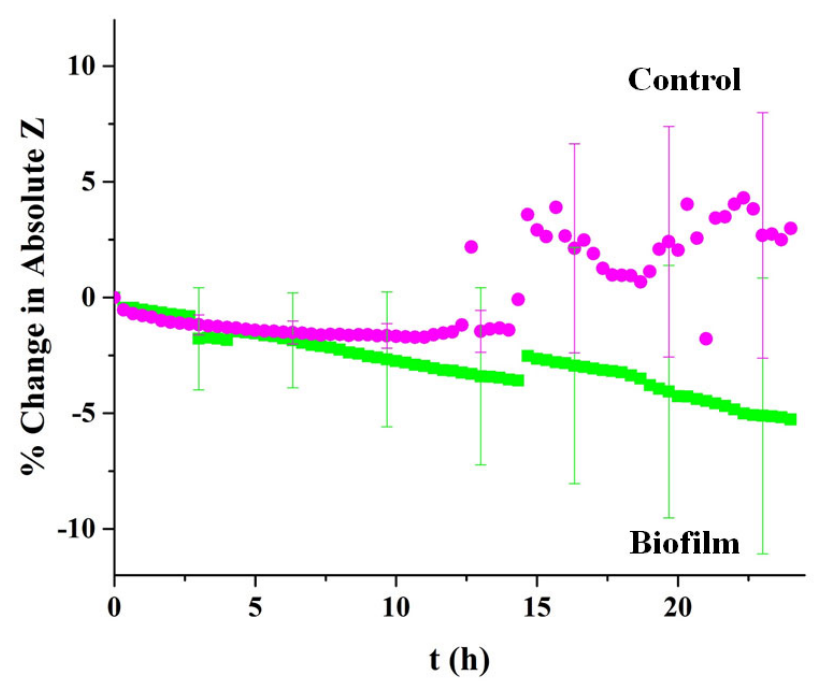

Figure 5: Average real-time impedance sensing results over the course of the 24-hour growth period, measured using the AIC. The pink circles correspond to control samples without biofilm and the green squares correspond to samples with biofilm formation. The excitation signal used was AC $200 \mathrm{mV}$ at $1 \mathrm{kHz}$, chosen to optimize sensitivity. The error bars denote standard error $(N=3)$.

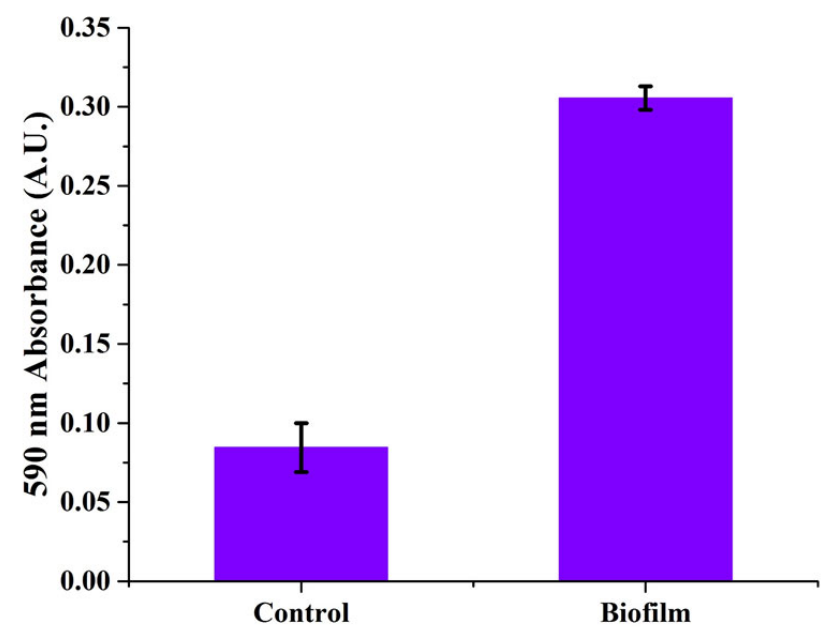

Figure 6: End-point biomass quantification for samples with and without biofilm measured with the AIC. Error bars correspond to standard error $(N=2)$.

These samples displayed a significant increase in biomass associated with the formation of biofilm compared to biofilm-free controls, similar to the potentiostat measured samples. The increased absorbance was statistically significant compared to LB media alone according to one-way ANOVA $(p<0.05)$. This indicates that the decrease in impedance is correlated with an increase in biomass. Essentially, the change that the AIC detects is related to increasing biofilm biomass on the FlexIS surface. This further confirms the viability of the FlexIS combined with the AIC for wireless biofilm monitoring.

Wireless monitoring was performed using the AIC interfaced with the BGM121 microcontroller. The impedance results for the FlexIS measured via wired UART and wireless Bluetooth are compared in Figure 7. The identical trend indicates that the FlexIS can be operated wirelessly without a decrease in performance. 


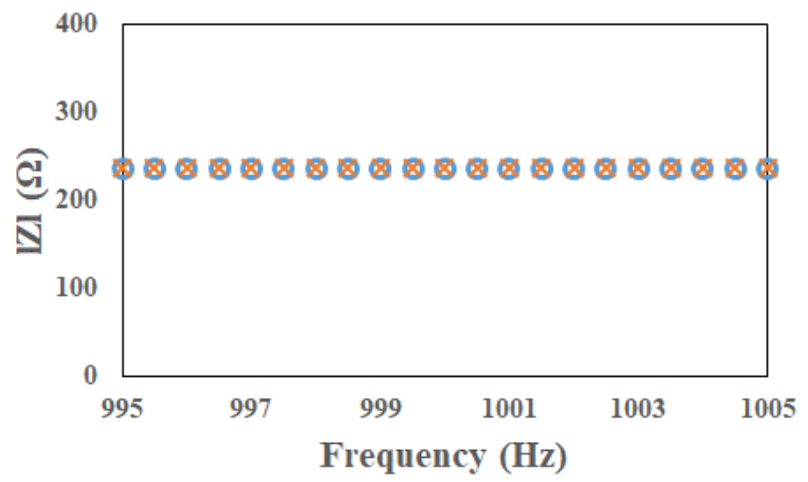

Figure 7: Frequency sweeps from 995-1005 Hz with wired (orange crosses) and wireless (blue circles) connections showing identical impedance responses from the FlexIS with growth media.

\section{CONCLUSIONS}

In this work, we have demonstrated the feasibility of the FlexIS as a platform for real-time, in situ monitoring of biofilm formation on catheters. The sharp decrease in impedance is correlated with a statistically significant increase in biofilm biomass. Successful implementation of this platform relies on its ability to operate in situ without impeding regular operation of the vulnerable device; in this case the flexible substrate allows the FlexIS to conform to the interior cylindrical lumen of a catheter and report on the level of biofilm colonization at the surface.

Initial characterization of this platform utilized a benchtop potentiostat for performing impedance measurements. However, bulky wired equipment is impractical for applications on inserted medical devices. Here, we demonstrate biofilm detection using the AIC to overcome this challenge. The AIC can be programmed with a BGM121 Bluetooth microcontroller to run impedance measurements and transmit the data wirelessly. The AIC was demonstrated as a viable approach for measuring biofilm formation with the FlexIS. The microcontroller measured a decrease in impedance with biofilm formation which correlated with an increase in biomass. Compared with the potentiostat standard, the AIC demonstrated reduced sensitivity. Nonetheless, the FlexIS was demonstrated as a viable tool for biofilm detection on catheter surfaces. This approach can be extended in the future to other vulnerable domains with complex, 3D geometry. Furthermore, the wireless control allows clinicians and researchers to read out data seamlessly without relying on bulky and wired equipment.

\section{ACKNOWLEDGEMENTS}

The authors would like to acknowledge the support of the Maryland Nanocenter and its FabLab for support in fabrication.

\section{REFERENCES}

[1] J.W. Costerton, P.S. Stewart, and E.P. Greenberg, "Bacterial biofilms: a common cause of persistent infections", Science, 284, 1318 (1999).

[2] A. Vendeville, K. Winzer, K. Huerlier, C.M. Tang, and K.R. Hardie, "Making "Sense" of Metabolism: Autoinducer-2, LUXS and Pathogenic Bacteria", Nat. Rev. Microbiol., 3, 383 (2005).

[3] H. Anwar, M.K. Dasgupta, and J.W. Costerton, "Testing the Susceptibility of Bacteria in Biofilms to Antibacterial Agents", Antimicrob. Agents Chemother., 34, 2043 (1990).

[4] J.W. Warren, "Catheter-Associated Urinary Tract Infections", Int. J. Antimicrob. Agents, 17, 299 (2001).
[5] D.G. Maki, C.E. Weise, and H.W. Sarafin, "A Semiquantitative Culture Method for Identifying Intravenous-Catheter-Related Infection", N. Engl. J. Med., 296, 1305 (1977).

[6] E. Peeters, H.J. Nelis, and T. Coenye, "Comparison of Multiple Methods for Quantification of Microbial Biofilms Grown in Microtiter Plates", J. Microbiol. Methods, 72, 157 (2008).

[7] R.J. Palmer and C. Sternberg, "Modern Microscopy in Biofilm Research: Confocal Microscopy and Other Approaches”, Curr. Opin. Biotechnol., 10, 263 (1999).

[8] Y.W. Kim, M.P. Mosteller, M.T. Meyer, H. Ben-Yoav, W.E. Bentley, and R. Ghodssi, "Microfluidic biofilm observation, analysis and treatment (MICRO-BOAT) platform", Technical Digest of the 2012 Solid-State Sensor and Actuator Workshop, Hilton Head Isl., SC, 6/3-7/12, Transducer Research Foundation, Cleveland (2012), pp. 233 - 236.

[9] K. Waszczuk, G. Gula, M. Swiatkowski, J. Olszewski, W. Herwich, Z. Drulis-Kawa, J. Gutowicz, and T. Gotszalk, "Evaluation of Pseudomonas aeruginosa biofilm formation using piezoelectric tuning fork mass sensors", Sens. Actuators, B, 170, 7 (2012).

[10] V. Reipa, J. Almeida, and K.D. Cole, "Long-term monitoring of biofilm growth and disinfection using a quartz crystal microbalance and reflectance measurements", J. Microbiol. Methods, 66, 449 (2006).

[11] Y.W. Kim, S.E. Sardari, M.T. Meyer, A.A. Iliadis, H.C. Wu, W.E. Bentley, and R. Ghodssi, "An ALD aluminum oxide passivated Surface Acoustic Wave sensor for early biofilm detection", Sens. Actuators, B, 163, 136 (2012).

[12] J. Paredes, S. Becerro, F. Arizti, A. Aguinaga, J.L. Del Pozo, and S. Arana, "Real time monitoring of the impedance characteristics of Staphylococcal bacterial biofilm cultures with a modified CDC reactor system", Biosens. Bioelectron., 38,226 (2012).

[13] X. Munoz-Berbel, F.J. Munoz, N. Vigues, and J. Mas, "On-chip impedance measurements to monitor biofilm formation in the water distribution network", Sens. Actuators, B, 118, 129 (2006).

[14] S. Subramanian, E.I. Tolstaya, T.E. Winkler, W.E. Bentley, and R. Ghodssi, "An Integrated Microsystem for Real-Time Detection and Threshold-Activated Treatment of Bacterial Biofilms", ACS Appl. Mater. Interfaces, 9, 31362 (2017).

[15] R.C. Huiszoon, P. Ramiah Rajasekaran, S. Subramanian, W.E. Bentley, and R. Ghodssi, "Flexible Impedance Sensor for In Situ Sensing of Catheter Biofilms", The $21^{\text {st }}$ International Conference on Miniaturized Systems for Chemistry and Life Sciences (MicroTAS 2017), Savannah, GA, 10/22-26/17, The Chemical and Biological Microsystems Society, Washington (2017), pp. $130-131$.

[16] R.C. Huiszoon, P. Ramiah Rajasekaran, W.E. Bentley, and R. Ghodssi, "Flexible Dual-Function Platform For In Situ Monitoring and Treatment of Bacterial Biofilms", The $9^{\text {th }}$ International Conference on Microtechnologies in Medicine and Biology (MMB 2018), Monterey, CA, 3/26-28/18, Transducer Research Foundation, San Diego (2018).

[17] K. Ong, J. Wang, R.S. Singh, L.G. Bachas, and C.A. Grimes, "Monitoring of Bacteria Growth Using a Wireless, Remote Query Resonant-Circuit Sensor: Application to Environmental Sensing”, Biosens. Bioelectron, 16, 305 (2001).

\section{CONTACT}

*R. Ghodssi, tel: +1-301-405-8158; ghodssi@umd.edu 